\title{
Cogniton-based Enlightenment of Creative Thinking: Examplars in Computer Science
}

\author{
Zhi-Quan Cheng, Shiyao Jin \\ School of Computer, National University of Defense Technology, Hunan Province, China \\ Email: cheng.zhiquan@gmail.com
}

Received 2012

\begin{abstract}
It is reputed that "Genius is 1\% inspiration and 99\% perspiration”, but it can also be noted that "sometimes, $1 \%$ inspiration is more important than $99 \%$ perspiration." As this $1 \%$ is so important, can it be understood, and even learned? If so, how can cognition be used to enlighten a scientist's inspiration (creative thinking)? Both questions are considered on the basis of cognitive theory in the paper. We illustrate our ideas with examples from computer science.
\end{abstract}

Keywords: Creative Thinking; Enlightenment; Cognition; Computer Graphics, Computer Simulation

\section{Introduction}

"Genius is one percent inspiration and ninety-nine percent perspiration, but sometimes, one-percent inspiration is more important than ninety-nine percent perspiration” is a quote usually attributed to Edison, when discussing his remarkable achievements. Generally, the later part of this saying is neglected when quoted, as the goal is to encourage hard work, rather than to point out the key role of distinguished scientists, like Edison, as a creative elite.

Scientific research, searching for new knowledge, appeals especially to individual creative people. Edward De Bono (De Bono, 2008), the father of creative thinking, suggested that creativeness is a particular way of thinking, and postulated that there are some basic principles and mental techniques that are commonly used while being creative. 150 years ago, Claude Bernard, the great French physiologist said (Bernard, 1865): "The genius of inventiveness maybe diminished or even smothered by a poor method, while a good method may increase and develop it... In biological science, the role of method is even more important...". These statements argue that the one-percent perspiration can be understood, and even learnt, in some way.

In our paper, using cognitive theory (Bermúdez, 2010), we explore how to understand creativity, and enlighten researchers in creative thinking (Sternberg, 2006). Our arguments are mainly addressed by using advances in computer science as exemplars, particularly in the areas of computer graphics and simulation. We explore creative habits of mind, and try to catch the insights how to generally improve one's creative thinking abilities, and how to apply them to new situations. Our work is carrying out at the difficult state of traditional methods pausing for about a decade (Mumford, 2003), and try to deal with it with new progress of computer science.

\section{Cognition and Creative Thinking for Scientists}

De Bono (De Bono, 2008) stated: "Creative thinking is not a talent, it is a skill that can be learnt. It empowers people, adding strength to their natural abilities, which improves teamwork, productivity and where appropriate, profits”. For a senior scientist, mental processes are the essence and the engine of creative endeavor. When a mind containing a wealth of information contemplates a problem, relevant information readily comes to into focus during thinking. A critical issue in problem solving is deciding whether the available information is sufficient or not. Since the information available in the mind must be recognized, we address the relationship between cognition and creative thinking, particularly for scientists.

\section{Cognition and Creativity Revisited}

The cognition (Kozbelt, Beghetto, \&Runco, 2010) that gives rise to creative thinking is not a single process or operation (Smith \& Ward, 1995), but rather consists of many different cognitive structures and processes that collaborate in a variety of ways to construct different types of creative output. There are two contrasting approaches to creativity in cognitive psychology. P. J. Guilford (Guilford, 1950) believed that creativity can be measured in terms of divergent production, or the number of varied responses made to specific tests. Rather than one good answer or single solution, divergent production results in many possible ideas. However, sheer number of possible ideas does not guarantee that they are useful, high quality and novel. The second approach is Sternberg and Lubart's investment theory of creativity (Sternberg \& Lubart, 1996). This theory states that the appropriate attributes for creativity are knowledge, an encouraging environment, an appropriate personality, intelligence, motivation and an appropriate thinking style.

Studies of creativity and cognition results (in terms of general intelligence) have found modest correlation between them (Silvia, 2008). Some researchers believe that creativity is the outcome of the same cognitive processes as intelligence, and only judge creativity in terms of its consequences. Recent advances in neural science further show that general intelligence reflects the combined performance of brains systems (Gläscher et al., 2010), but the brain is still a functional black box, in terms of how cognitive processes produce something novel.

In recent years, two approaches have dominated the research literature on cognition-based creativity: process-oriented mod- 
els of creativity, and systems-oriented models. Process-oriented models concentrate on cognitive aspects of creativity; while systems-oriented models take a broader approach to creativity involving non-cognitive factors as well as cognitive ones. We suggest a process-oriented model, which we suggest simulates how the cognitive process relates to creativity.

\section{Framework of Mental Cognition}

We firstly recall how cognition works, before it acts as a stimulus for creativity.

When one thinks of Einstein, it is natural to assume that his brain differed from that of the average person. In 1999, an anatomical study was made of Einstein's brain. Interestingly, his brain was smaller than average. However, the study (Witelson et al., 1999) also found that Einstein's parietal lobes were 15\% wider than average. Science now points out that these lobes are usually connected to spatial and visual cognition, as well as to mathematics. Of course, the brain is a complex and still-mysterious organ, but it may be that we can glean some additional insight from this study: the relation of cognition to creativity has a physiological basis.

In psychology, a cognitive process refers to how people perceive, remember, think, speak, and solve problems. The cognitive approach was brought to prominence by Donald Broadbent (Broadbent, 1958), who put forward an information processing model of cognition. This is a way of thinking and reasoning about mental processes, envisioning them as akin to software running on a computer that is the brain. Theories refer to forms of input, representation, computation or processing, and outputs. Because of the use of computational metaphors and terminology, cognitive psychology was able to benefit greatly from the flourishing of research in computer science.

Based on such an information processing model of cognition, we illustrate the cognition framework (Figure 1) using recent conceptual terms. The terms describe input sensations and perception, output behavior, intrinsic and learning cognition function units, and main memory. Memories (Atkinson \& Shiffrin, 1968) may be stored in long-term memory (LTM), short-term memory (STM), autobiographical memory (ABM), and sensory memory (SM).

- SM. Sensory memory corresponds approximately to the initial 200-500 milliseconds after an item is perceived. The ability to look at an item, and remember what it looked like within just a second of observation, is an example of sensory memory.

- STM. Short-term memory allows recall for a period of several seconds to a minute without rehearsal. It provides the ability to hold a small amount of information in mind in an active, readily available state for a short period of time. The duration of short-term memory (when rehearsal or active maintenance is prevented) is believed to be of the order of seconds. A commonly cited capacity is $7 \pm 2$ stored items.

- LTM. Long-term memory is memory in which associations among items are stored, according to the dual-store memory model (Atkinson and Shiffrin, 1968). Memories can reside in the short-term "buffer" for a limited time while they are simultaneously strengthening their associations in long-term memory.

- $A B M$. Autobiographical memory is a memory system consisting of episodes recollected from an individual's life, based on a combination of episodic (personal experiences and specific objects, people and events experienced at par- ticular time and place) and semantic (general knowledge and facts about the world) memory (Williams, Conway, \& Cohen, 2008).

We suggest a model to mental cognition using an analogy to the Von Neumann architecture (Neumann, 1945) from computer science. This model is not meant to be a serious suggestion of how the brain works, but rather, a simplified description which is adequate for the purposes of discussing cognition and creativity.

The correspondences between mind and computer could be can be considered to be: input devices to input sensory and perceptual organs, processor to intrinsic and learning cognition, main memory to STM, disk to LTM, output devices to output motor and behavior organs, input channels to $S M$ and output channels to $A B M$. See Figure 1.

Using this model, the mental cognitive process can be described as follows:

1) The recognition and understanding of events, objects, and stimuli through the use of senses (sight, hearing, touch, etc.). Several different types of perception exist, and the data merged to give the input.

2) The mind performs intrinsic cognition as primary processing of the input data, then more deeply operates on the data using learned cognition.

3) Operations are performed by retrieving stored information in response to cues, enable the information to be used in multiple processes or activities.

4) Learned information is stored in the STM or LTM according to judgment, and if necessary, appropriate behaviors are output.

Clearly, our framework of mental cognition is a storedmemory model. The memory is the unit in which information is encoded, and stored, and from which it is retrieved. To sum up, information results from cognition of reality.

\section{Correlation between Cognition and Creativity}

The correlation between cognition and creativity is an important problem in philosophy and psychology. We must consider the relationship, its origins and its forms, as well as the principles and laws of cognitive activity, and its development. As a selective reflection of the world, cognition and filtering of information underpins human reasoning and drives human achievement.

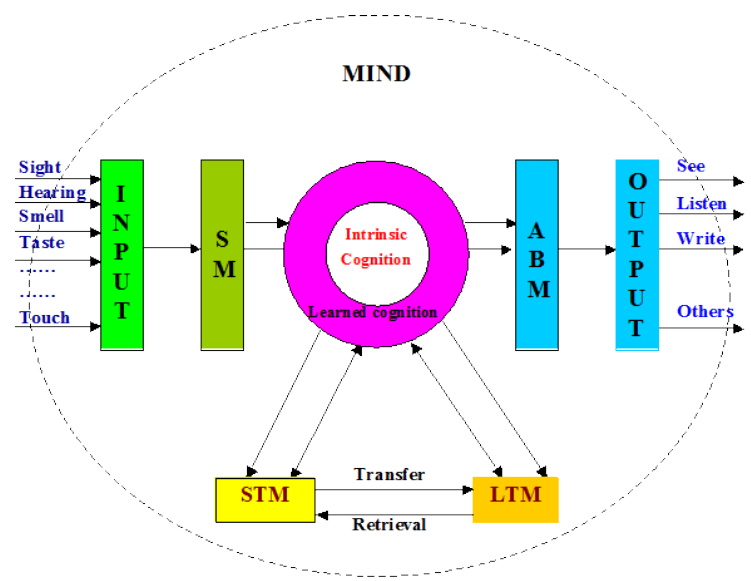

Figure 1.

Framework of mental cognition. 
There is much truth in the saying that in science the mind of the scientist can build only as high as the foundations constructed by existing information will support. One of the research worker's duties is to follow the scientific literature, but learning needs to be done with a critical, reflective attitude if originality and freshness of outlook are not to be lost. Merely to accumulate information as a sort of capital investment is insufficient.

It is usual to carefully gather information dealing with the particular problem on which one is going to work. However, surprising as it may seem at first, some scientists consider that this is unwise. They contend that investigating what others have said on the subject conditions the mind to see the problem in the same way and make it difficult to find a new and fruitful approach. There are even some grounds for discouraging an excessive amount of reading in the general field of science in which one is going to work. Many successful investigators were not trained in the branch of science in which they made their most brilliant discoveries. But these researchers still had relevant knowledge and were well trained. The same dilemma faces all creative workers.

We may analyze this observation further. When a mind containing a wealth of information contemplates a problem, relevant information provides useful cues to the solution. It is advisable to make a thorough study of all the relevant literature early in the investigation, for much effort may be wasted if even only one significant article is missed. However, if that information is insufficient, then the mass of this information makes it more difficult for the mind to conjure up original ideas. Further, some of that information maybe actually inappropriate, in which case it presents a more serious barrier to new and productive ideas. During the course of an investigation, as well as watching for new papers on the problem, it is very useful to read more generally over a wide field keeping a constant watch for some new principle or technique that may be useable. Often, taking or adapting existing ideas from a different area is a key problem solving step.

The best way of meeting the dilemma of "knowing too much" is to critically obtain information, striving to maintain independence of mind and avoid becoming conventionalized. Francis Bacon said: "Read not to contradict and confute, nor to believe and take for granted...but to weigh and consider". The scientist with the right outlook for research develops a habit of correlating what is read with his knowledge, looking for significant analogies and generalizations.

\section{Simulation of Cognition and Creative Thinking}

In his pioneering work Art of Thought, Wallas (Wallas, 1926) presented one of the first models of the creative process. In the Wallas stage model, creative insights and illuminations may be explained by a process comprising 5 stages:

1) Preparation. The scientist focus his mind on the problem and explores the problem's dimensions;

2) Incubation. The problem is internalized into the unconscious mind and nothing appears externally to be happening;

3) Intimation. The creative person gets a feeling that a solution is on its way;

4) Illumination or insight. The creative idea bursts forth from its preconscious processing into conscious awareness;

5) Verification. The idea is consciously verified, elaborated, and then applied.
Wallas' model is often treated as four stages, with intimation seen as a sub-stage. Wallas considered creativity to be a legacy of the evolutionary process, which allowed humans to quickly adapt to rapidly changing environments. The implied theory behind Wallas' model-that creative thinking is a subconscious process that cannot be directed, and that creative and analytical thinking are complementary-is reflected to varying degrees in other models of creativity. In contrast to the prominent role that some models give to subconscious processes, Perkins (Perkins, 1981) argues that subconscious mental processes are behind all thinking and, therefore, play no extraordinary role in creative thinking. (Ram et al., 1995) proposed the five components for creativity: inferential mechanisms, knowledge sources, tasks, situation, and strategic control.

While there are many models for the process of creative thinking, it is not difficult to see consistent themes that span them all. 1) The creative process involves purposeful analysis, imaginative idea generation, and critical evaluation-the overall creative process is a balance of imagination and analysis. 2) Older models tend to imply that creative ideas result from subconscious processes, largely outside the control of the thinker. Modern models tend to imply purposeful generation of new ideas, under the direct control of the thinker. 3) The overall creative process requires a drive to action and the implementation of ideas. We must do more than simply imagine new things, we must work to make them concrete realities.

These insights from a review of the many models of creative thinking have encouraged us to produce a synthetic simulation model (Humphreys, 2004) of creative thinking that combines the concepts behind the various models proposed over the last years.(Figure 2)

Our model has three main components as follows:

- Recognition. Recognition uses memories storing information in SM, STM, and LTM, sensing and learning functional organs, and cognition processors.

- Creativity. Creativity units (including creative thinking mechanisms) and skills (creativity mapping) work together to produce novel and useful produces (Mumford, 2003). The dominant factors are usually identified as "the four Ps"-process, product, person and place (Kozbelt, 2010). A focus on process is shown in cognitive approaches that try to describe thought mechanisms and techniques for creative

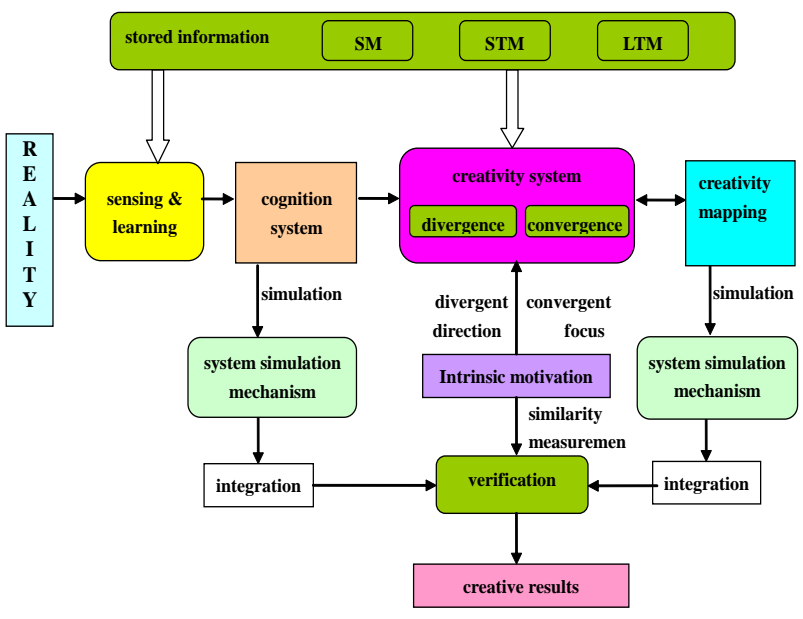

Figure 2.

Simulation model of cognition and creativity. 
thinking. Theories invoking divergent rather than convergent thinking (such as Guiford), or those describing the staging of the creative process (such as Wallas) are primarily theories of creative process. J. P. Guilford (Guiford, 1967) performed important work in the field of creativity, drawing a distinction between convergent and divergent production or thinking. Convergent thinking involves aiming at a single, correct solution to a problem, whereas divergent thinking involves creatively generating multiple answers to a problem. Divergent thinking is sometimes used as a synonym for creativity in the psychology literature. Intrinsic, task-focused motivation is also essential to creativity.

- Verification. After verifying, elaborating, and applying the creative idea using similarity, a creative (original and worthwhile) result is produced.

Note that the main characters of this model are the simulation factors, which are seamlessly integrated into the mechanical analysis of cognition and creativity. By using the computer simulation units, we provide a foundation to simulate the abstract mental model of cognition and creativity. The simulation could be performed by finding analytical solutions to cognition-based creative thinking problems, which enables the recording, verification, and even prediction of the behavior of the cognition-based creativity from a set of parameters and initial conditions. Furthermore, by concurrently performing simulation and real cognition and creativity tasks, our new framework can effectively deal with the interplay between experiment, simulation, and theory for the cognition and creativity correlation investigation.

Our work continues in the tradition of others (e.g. (Graham-Rowe, 2005)) in asserting that creativity is a balance of imagination and analysis by using information. The simulation model also purposefully avoids taking a stand on the controversy of whether creativity is a conscious or subconscious cognitive result. While we personally believe that intrinsic motivation is a conscious, non-magical mental action, the activity of "producing creative results" in the model accepts creative ideas regardless of their source. Finally, note that this model clearly supports the notion that creativity is a step beyond the simple recognition of reality. The simulation model has value only when it is implemented in the real world.

\section{Creative Thinking Enlightenment}

As it is still impossible to physically record the mental cognition and creativity process, we use our former model to simulate the functionalities of learning and thinking. In the section, we first review thinking, and discuss why visual analogical thinking is an appropriate choice for enlightening creative thinking. We then consider an example from computer graphics of automatic 3D model creation.

\section{Thinking Mechanism Review}

Reasoning, visual thinking, intuition and inspiration are standing thinking mechanisms. In the following, we discuss which can be learned and are applicable for a scientist performing creative research.

The origin of creativity is somewhat beyond the reach of logical reasoning (Aldo, 2003). The role of logical reasoning in research is not in making discoveries (either factual or theoretical), but verifying, interpreting and developing them and building a general theoretical scheme. Most scientific facts and theories are only true under certain conditions and our knowledge is so incomplete that at best we can only reason based on probabilities and possibilities. Besides logical reasoning, analogical reasoning is a mutually exclusive alternative for the thinking. Analogs are achieved by a comparison that determines the degree of similarity, or an inference based on resemblance or correspondence. As we know, while results from an analogy may or may not be true, analogical thinking can produce new ideas.

Visual thinking, or right brained thinking, is the common phenomenon of thinking through visual processing using the part of the brain that is emotional and creative to organize information in an intuitive and simultaneous way. During his lifetime, Einstein often claimed that he thought in images and sensations rather than in words.

Intuition and inspiration indicate a sudden enlightenment or comprehension of a situation, a clarifying idea which dramatically springs into the consciousness, often, though not necessarily, when one scientist is not consciously thinking of special subject. The most characteristic circumstances of an intuition are a period of intense work on the subject accompanied by a desire for its solution, followed by the appearance of the creative idea with dramatic suddenness and often a sense of certainty. Intuition is still a mystical issue, and we are a long way from really understanding and simulate it.

Theobald Smith's said that: "Discovery should come as an adventure rather than as the result of a logical process of thought. Sharp, prolonged thinking is necessary that we may keep on the chosen road, but it does not necessarily lead to discovery”. As we know, all scientific advances rest on a base of previous knowledge. Often, the application or transfer of a new principle or technique from another field provides the central idea upon which an investigation hinges. Such transfer is a typical analogical thinking scheme. In attempting to apply an existing technique to a new problem, some new knowledge arises.

In the process of creativity, it is not the knowledge (information) stored which is so important as the scientist making use of knowledge. During scientific creative thinking, analogical and visual thinking are both learnable and applicable tactics.

\section{New 3D Model Creation}

3D modeling is the process of developing a mathematical representation of any three-dimensional object, called a "3D model”. It is one of the most fundamental tasks in computer graphics. We demonstrate how analogical and visual thinking tactics may be employed within a computer program to automatically creatively generate novel 3D models.

Creating a 3D model of modest complexity is typically a daunting task, so a sensible strategy is to generate a novel shape as a variation of one or more existing models. In a typical paper (Xu et al., 2010), new shapes are synthesized replicating a certain style extracted from a set of input shapes. The particular style studied is the anisotropic scaling of the shape parts. The key enabling concept is style-content separation which facilitates the computation of part correspondence across a whole set of input shapes exhibiting large style variations. Style-content separation then allows style transfer as a basis for synthesis of new objects. Figure $\mathbf{3}$ show the style-content separation process and automatic 3D model creation. Our idea is a typical example of the use of analogical thinking, this time performed 
b

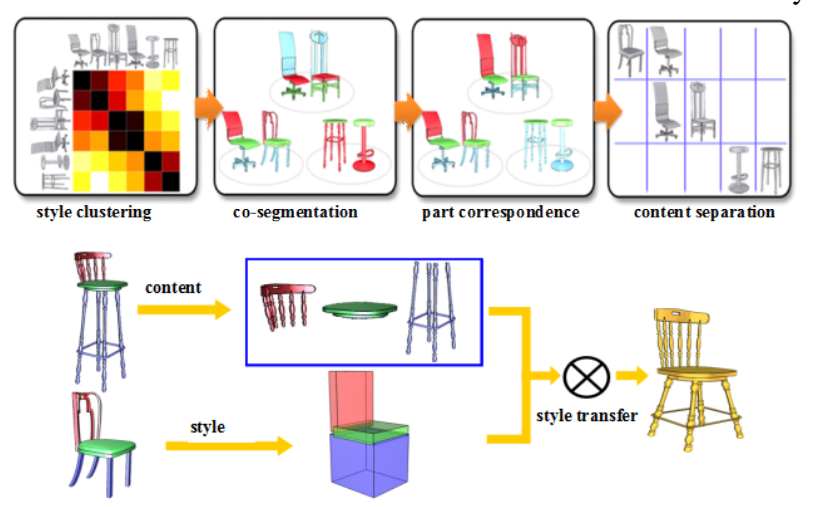

Figure 3.

Automatic 3D model creation. Up: the process of content-style separation, bottom: new model creation by style transfer.

the computer, to create different styles of model. Using the transfer rule, some newly-created models do not meet or requirements and expectations. This shows that when concepts are transferred to another area, they are often instrumental in uncovering still further knowledge. The example gives some hints on how best to go about various activities that constitute research, but explicit rules can not be laid out since research is an investigatory art.

The possibility of developments in the transfer method is perhaps the main reason why the scientist needs to keep himself informed of at least the principal developments taking place in more than his own narrow field of work.

\section{Conclusions}

A scientist works like a pioneer as he explores the frontier of knowledge, and requires many of the same attributes: enterprise and initiative, readiness to face difficulties and overcome them with his own resourcefulness and ingenuity, perseverance, a spirit of adventure, a certain dissatisfaction with well-known territory and prevailing ideas, and an eagerness to try his own judgment. What is produced can come in many forms and is not specifically singled out in a subject or area.

In this paper, we have tried to suggest how cognition works for creative thinking, which is more important than the $99 \%$ perspiration. We have tried to solve the problem by using exemplars from computer science. Firstly, we have made use of computer simulation to investigate the correlation between cognition and creative thinking. Then, the 3D model creation in computer graphics is used as an illustration to explain why the analogical and visual thinking are enlightening for creative thinking.

It is probably inevitable that any paper which attempts to deal with such a wide and complex subject will have many limitations. We hope the shortcomings of our work may provoke others whose achievements and experience are greater than ours to write about this subject and so build up a greater body of organized knowledge than is available in the literature at present.

\section{Acknowledgements}

The work is funded by the NSFC of China (No. 61103084 and 61272334) and NUDT Education Reforming grants.

\section{REFERENCES}

De Bono, E. (2008). How to have creative idea: 62 games to develop the mind. Publisher: Vermilion.

Bernard, C. (1865). An introduction to the study of experimental medicine (English translation). Macmillan \& co. New York, 1927.

Guilford, J. P. (1950). Creativity. American Psychologist, 5(9), 444-454.

Sternberg, R. J., \& Lubart, T. I. (1996). Investing in creativity. American Psychologist, 51(7), 677-688.

Silvia, P. J. (2008). Creativity and intelligence revisited: A reanalysis of Wallach and Kogan (1965). Creativity Research Journal, 20, 34-39.

Gläscher J., Rudrauf D., Colom R., Paul L. Tranel K., D., Damasio H., \& Adolphs R. (2010). The distributed neural system for general intelligence revealed by lesion mapping. In Proceedings of the National Academy of Sciences.

Witelson S. F., Kigar D. L., \& Harvey T. (1999). The exceptional brain of Albert Einstein. Lancet, 353, 2149-2153.

Broadbent, D. E. (1987). Perception and communication. Oxford: Oxford University Press.

von Neumann, J. (1945). First Draft of a Report on the EDVAC.

Mooney G. A., Fewtrell R. F., \& Bligh J. G. (1999). Cognitive process modelling: computer tools for creative thinking and managing learning. Medical Teacher, 21(3), 277-280

Wallas, G. (1926). Art of Thought.

Perkins, DN (1981) The Mind's Best Work. Cambridge, MA: Harvard University Press.

Ram A., Wills L., Domeshek E., Nersessian N., \&Kolodner J.(1995). Understanding the Creative Mind. AI Journal, 79, 111-128.

Sternberg, R.J. (2006). The Nature of Creativity. Creativity Research Journal, 18(1), 87-98.

Gabora, L. (2002). Cognitive mechanisms underlying the creative process. Proceedings of the Fourth International Conference on Creativity and Cognition (pp. 126-133), UK.

Mumford, M. D. (2003). Where have we been, where are we going? Taking stock in creativity research. Creativity Research Journal, 15, 107-120.

Kozbelt, A., Beghetto, R. A. \& Runco, M. A. (2010). Theories of Creativity. The Cambridge Handbook of Creativity. Cambridge University Press.

Williams, H. L., Conway, M. A., \& Cohen, G. (2008). Autobiographical memory. Memory in the Real World (3rd ed., pp. 21-90). Hove, UK: Psychology Press.

Atkinson, R.C.; Shiffrin, R.M. (1968). Human memory: A proposed system and its control processes. In Proceedings of The psychology of learning and motivation (2, pp. 89-195). New York: Academic Press.

Humphreys P. (2004). Extending Ourselves: Computational Science, Empiricism, and Scientific Method. Oxford: Oxford University Press.

Graham-Rowe, D. (2005). Mission to build a simulated brain begins. NewScientist.

Aldo A., Lex W., \& Ganter B.. (2003) Conceptual Structures for Knowledge Creation and Communication, LNAI 2746, 16-36.

Xu K., Li H., Zhang H., Cohen-Or D., Xiong Y., \& Cheng Z.-Q. (2010). Style-Content Separation by Anisotropic Part Scales. ACM Transactions on Graphics, 29(6), 184:1-184:10.

Bermúdez. J. L. (2010). Cognitive Science: An Introduction to the Science of the Mind. Publisher: Cambridge University Press. 